T. Nakano

Nagoya Math. J.

Vol. 116 (1989), 139-148

\title{
REGULAR ACTIONS OF SIMPLE ALGEBRAIC GROUPS ON PROJECTIVE THREEFOLDS
}

\author{
TETSUO NAKANO
}

\section{$\S 1$. Introduction}

The purpose of this note is to study regular actions of simple algebraic groups on projective threefolds as an application of the theory of algebraic threefolds, especially Mori Theory and the theory of Fano threefolds (cf. Mori [11], Iskovskih [7, 8]). The motivation for this study is as follows. In a series of papers, Umemura, in part jointly with Mukai, has classified maximal connected algebraic subgroups of the Cremona group of three variables and also constructed minimal rational threefolds which correspond to such subgroups (cf. Umemura [16-19], Mukai-Umemura [12]). In particular, Umemura and Mukai studied in [12] the $\boldsymbol{S L}(2, C)$-equivariant smooth projectivization of $\boldsymbol{S L}(2, C) / G$, where $G$ is a binary icosahedral or octahedral subgroup of $S L(2, C)$. The study of equivariant smooth projectivization of $S L(2, C) / G$ for any finite subgroup $G$ has been completed along their lines in Nakano [14]. The main trick of these studies is the investigation of equivariant contraction maps of extremal rays in the context of Mori Theory [11]. In this note, we apply a similar idea to projective threefolds with a regular action of a simple algebraic group and determine which simple algebraic groups can act regularly and nontrivially on projective threefolds and in which fashion. We also need some standard (but difficult) facts from the theory of Fano threefolds. For the precise statement, see Theorem 1 in the main text. For the proof of this theorem, we need a classification of closed subgroups of simple algebraic groups of codimension 1 and 2, which could be derived easily from the classical work of Dynkin [4]. However, we shall give a geometric proof independent of [4] which leads up directly to the proof of Theorem 1. On the whole, we shall establish by geometric methods the scarcity of closed subgroups of small codimension in simple algebraic groups, which is implied in Dynkin [4]. In the following, all the algebraic varieties and algebraic groups are assumed to be defined over a fixed

Received August 17, 1988. 
algebraically closed field $k$ of characteristic zero.

I am grateful to Professor T. Kambayashi for critically reading the manuscript of the present paper and offering useful pieces of advice for improvement. The contents of this paper constitute the second part of the author's doctoral dissertation, written under Professor Kambayashi's direction and submitted to Tokyo Denki University in 1988. The first part is contained in [14].

\section{§ 2. Statement and proof of the main result}

Let $G$ be a simple algebraic group and suppose that $G$ acts on a projective threefold $X$ regularly and nontrivially. If $\pi: \tilde{G} \rightarrow G$ is the universal covering map of $G$, then $\tilde{G}$ acts regularly and nontrivially on $X$ through $\pi$. Hence we may and shall always assume that the acting simple algebraic group is simply-connected without losing generality. Our main result is as follows.

ThEOREM 1. Let $G$ be a simply-connected, simple algebraic group. Then the following assertions hold:

(1) There exists a projective threefold $X$ on which $G$ acts regularly and nontrivially if and only if $G=S L(i)(i=2,3,4)$ or $S O(5)$.

(2) If $G=S L(4)$, then $X$ is isomorphic to the projective 3-space $P^{3}$. If $G=S O(5)$, then $X$ is isomorphic to $P^{3}$ or to a smooth quadric threefold $Q_{2}$ in $P^{4}$.

Remark 2. (i) In the case $G=S L(2)$, the classification of such $X$ 's has been made complete under the additional hypothesis that $X$ has an open dense orbit; see Mukai-Umemura [12] and Nakano [14]. As for the case $G=\boldsymbol{S} L(3)$, Mabuchi has classified completely the smooth projective threefolds with a nontrivial regular $S L(3, C)$-action (see [10]). (Actually, he classified more generally the smooth projective $n$-folds with a nontrivial regular $S L(n, C)$-action.)

(ii) In statement (2) of the theorem above, the $G$-actions on $X$ are quite natural ones. Specifics will be given in the proof below.

Proof of Theorem 1. We will prove two lemmas first. Although parts of the results of these lemmas are contained in the classical work of Dynkin [4], we shall give self-contained, geometric proofs that lead themselves directly to the proof of Theorem 1. 
LEMMA 3. Let $G$ be a simply-connected, simple algebraic group. Then the following assertions hold:

(1) There exists a closed subgroup $H \subset G$ of codimension 1 if and only if $G=S L(2)$.

(2) If $G=S L(2), B:=\left\{\left[\begin{array}{cc}a & b \\ 0 & 1 / a\end{array}\right] \mid a \in k^{\times}, b \in k\right\}$ is the unique closed subgroup of codimension 1 up to conjugation.

Proof. We will prove the "only if" part of (1) first. Let $H$ be a closed subgroup of $G$ such that $\operatorname{codim}_{G}(H)=1$. Then $G / H$ is an irreducible smooth quasi-projective curve (cf. Hochschild [6; Chapter XII, pp. 178]). Let $X$ be the smooth projectivization of $G / H$. Considering the induced $G$-action on the function field of $G / H$, we find that $G$ acts on $X$ regularly and $G / H$ is an open dense orbit of $X$.

Now, since $G / H$ is rational (cf. Mukai-Umemura [12; Lemma (1.15), pp. 503]), $X$ is isomorphic to $P^{1}$. Let $\varphi: G \rightarrow \operatorname{Aut}(X) \simeq P G L(2)$ be the homomorphism induced by the $G$-action on $X=\boldsymbol{P}^{1}$. If $(\operatorname{dim}(G)>\operatorname{dim}(\boldsymbol{P G L}(2))$ $=3$, then $G$ acts on $X=\boldsymbol{P}^{1}$ trivially, contradicting the fact that $X$ has an open orbit. So $\operatorname{dim}(G) \leq 3$ and $G$ must be $S L(2)$ if we count the dimensions of simple algebraic groups.

Next, if $G=S L(2)$, clearly $B$ as above is a closed subgroup of codimension 1. Let $H$ be any such subgroup. Then the argument in (1) shows that $G / H$ is the open orbit of $P^{1}$ equipped with a nontrivial $S L$ (2)-action. But the standard action of $S L(2)$ on $\boldsymbol{P}^{1}$ is the only nontrivial $S L(2)$-action on $\boldsymbol{P}^{1}, \varepsilon \mathbf{n}$ the isotropy group of a point with respect to this action is conjugate to $B$.

Q.E.D.

LEMMA 4. Let $G$ be a simply-connected, simple algebraic group. Then the following assertions hold:

(1) There exists a closed subgroup $H \subset G$ of codimension 2 if and only if $G=S L(2)$ or $S L(3)$.

(2) If $G=S L(2)$, then $H$ is conjugate to one and only one of the following closed subgroups:

$$
\begin{aligned}
& T=\left\{\left[\begin{array}{cc}
a & 0 \\
0 & 1 / a
\end{array}\right] \mid a \in k^{\times}\right\}, \\
& N(T)=\left\{\left[\begin{array}{cc}
a & 0 \\
0 & 1 / a
\end{array}\right] \mid a \in k^{\times}\right\} \cup\left\{\left[\begin{array}{cc}
0 & -b \\
1 / b & 0
\end{array}\right] \mid b \in k^{\times}\right\}, \\
& E_{n}=\left\{\left[\begin{array}{cc}
\zeta & a \\
0 & 1 / \zeta
\end{array}\right] \mid \zeta^{n}=1, a \in k\right\}, \quad n \geq 1 .
\end{aligned}
$$


(3) If $G=S L(3)$, then $H$ is conjugate to one and only one of the following closed subgroups:

$$
\begin{aligned}
& H_{+}=\left\{\left(a_{i j}\right)_{1 \leq i, j \leq 3} \mid a_{21}=a_{31}=0\right\} \\
& \left.H_{-}=\left(a_{i j}\right) \mid a_{32} a_{13}-a_{12} a_{33}=0, a_{12} a_{23}-a_{22} a_{13}=0\right\} .
\end{aligned}
$$

Proof. The "if" part of assertion (1) is clear in view of the subgroups explicitly listed in (2) and (3). Next, we will prove the "only if" part of (1). Let $H$ be a closed subgroup of $G$ of codimension 2. Then $G / H$ is a smooth quasi-projective surface. Hence, by Sumihiro [15], there exists a $G$-equivariant projective completion $i: G / H \longrightarrow X_{0}$, where $X_{0}$ is a projective surface with a regular $G$-action and $i$ is a $G$-equivariant open immersion. Let $f: X_{1} \rightarrow X_{0}$ be the normalization of $X_{0}$. Then $G$ acts on $X_{1}$ in such a way that $f$ is $G$-equivariant. The singular loci of $X_{1}$ is a finite set $\left\{P_{1}, P_{2}, \cdots, P_{r}\right\}$ and each $P_{j}$ is a fixed point of the given action. We blow-up $\left\{P_{1}, \cdots, P_{r}\right\}$ and let $\pi: X_{2} \rightarrow X_{1}$ be the blowing-up. Then $G$ acts on $X_{2}$ so that $\pi$ is $G$-equivariant. In this way, we repeat normalizations and blowing-ups of the singular loci until we finally get a smooth projective surface $X$ in finitely many steps (this is Zariski's way of resolution of surface singularities). Thus, we get a smooth projective G-equivariant completion $j: G / H \smile X$.

Now, we will consider $G$-equivariant contractions of extremal rays of $X$ (cf. Mori [11], Mukai-Umemura [12]).

Since $G / H$ is rational (cf. Mukai-Umemura [12; Lemma (1.15)]), $X$ is also rational and the canonical bundle of $X$ is not numerically effective. Hence, there exist an extremal ray $R$ and a resulting contraction map $\operatorname{cont}_{R}$, which turns out to be $G$-equivariant (cf. Mukai-Umemura [12; $\S 5$, pp. 513]). The structure of $f:=\operatorname{cont}_{R}:=X \rightarrow Y$ is classified into 3 cases (cf. Mori [11; Theorem (2.1), pp. 141]). If $f$ is a $G$-equivariant $P^{1}$-bundle over a smooth curve $Y$, or $X \simeq P^{2}$ and $Y=\operatorname{Spec}(k)$, then we stop. Note that in the former case, $Y \simeq \boldsymbol{P}^{1}$ because $Y$ has an open dense orbit which is rational. If $f: X \rightarrow Y$ is a $G$-equivariant blowing-up of a smooth projective surface $Y$, then $Y$ is again rational and $Y$ has an extremal rational curve. Applying the same process to $Y$, we finally get a $G$ equivariant morphism of $X$ onto $\boldsymbol{P}^{2}$ or $\boldsymbol{P}^{1}$. Since $G$ acts on $\boldsymbol{P}^{2}$ or $\boldsymbol{P}^{1}$ nontrivially, we get $\operatorname{dim} G \leq \operatorname{dim} \operatorname{Aut}\left(P^{2}\right)=8$. Therefore $G$ must be $S L(2)$ or $S L(3)$ if we count the dimensions of simple algebraic groups.

Let us now prove (2) and (3). Assume $G=S L(3)$. In this case, the 
proof of the "only if" part of (1) shows that $G / H$ has $P^{2}$ as a $G$-equivariant completion. Now, let $\alpha_{+}: \boldsymbol{S L}(3) \rightarrow \boldsymbol{G L}(3)$ be the natural 3-dimensional representation of $S L(3)$ and set $\alpha_{-}:=\left(\left(\alpha_{+}\right)^{t}\right)^{-1}$, the contragradient representation of $\alpha_{+}$. Then, as is well-known, $\alpha_{+}$and $\alpha_{-}$are all and only inequivalent 3-dimensional nontrivial representations of $\boldsymbol{S L}(3)$. Let $\boldsymbol{P}_{+}^{2}$ (resp. $\boldsymbol{P}_{-}^{2}$ ) be the $\boldsymbol{P}^{2}$ with an induced $\boldsymbol{S} \boldsymbol{L}(3)$-action from $\alpha_{+}$(resp. $\alpha_{-}$). $\boldsymbol{P}_{+}^{2}$ and $\boldsymbol{P}_{-}^{2}$ are all the nontrivial $\boldsymbol{S L}(3)$-actions on $\boldsymbol{P}^{2}$ up to $\boldsymbol{S L}(3)$-equivariant isomorphisms. Now, $\boldsymbol{P}_{+}^{2}$ and $\boldsymbol{P}_{-}^{2}$ are homogeneous spaces of $\boldsymbol{S L}(3, \boldsymbol{C})$ and $\boldsymbol{P}_{+}^{2} \simeq \boldsymbol{S L}(3, \boldsymbol{C}) / H_{+}$and $\boldsymbol{P}_{-}^{2} \simeq \boldsymbol{S L}(3, \boldsymbol{C}) / H_{-}$. Hence $H$ is conjugate to either $H_{+}$or $H_{-}$.

Assume $G=S L(2)$. Then the proof of the "only if" part of (1) shows that $G / H$ has either $P^{2}$ or the Hirzebruch surface $F_{n}:=P\left(\mathcal{O}_{P_{1}} \oplus \mathcal{O}_{P^{1}}(-n)\right)$ $(n \geq 0)$ as a $G$-equivariant completion. An easy analysis of the $S L(2)$ actions on these surfaces gives the result. We omit the details since we do not need this part in the proof of Theorem $1 . \quad$ Q.E.D.

We now turn to the proof of statements (1) and (2) of Theorem 1. The "if" part of (1) is clear. In fact, if $G=\boldsymbol{S L}(i)(i=2,3,4)$ or $\boldsymbol{S O}(5)$, then it is easy to see that $G$ can act nontrivially on $P^{3}$. Let us prove the "only if" part of (1) and (2) at the same time. Let $G$ be a simplyconnected, simple algebraic group and suppose that $G$ acts on a projective threefold $X$ regularly and nontrivially. We assume $G \neq S L(2), S L(3)$ from now on.

Let $\pi: \tilde{X} \rightarrow X$ be a resolution of singularities by Hironaka [5]. From Lemma 3 and Lemma 4, we know that every point of $\operatorname{Sing}(X)(:=$ the singular locus of $X$ ) is a fixed point $\operatorname{since} \operatorname{Sing}(X)$ is a $G$-stable subset. Therefore, in the resolution process of $X$, the center of each blowing-up is $G$-stable. Hence we have a regular nontrivial action of $G$ on $\tilde{X}$ such that $\pi$ is $G$-equivariant. Now, we have the following lemma, whose proof has been simplified to its present form owing to a suggestion of Spencer Bloch. (A similar argument is found in Bialynicki-Birula [1].)

LEMMA 5. Let $G$ be a simply-connected, simple algebraic group which is different from $S L(2)$ and $S L(3)$. Assume that $G$ acts regularly on a projective threefold $Z$ and that $x \in Z$ is a fixed point of $G$. If $Z$ is smooth at $x$, then this action is trivial.

Proof. We consider the induced linear representation of $G$ on the 3-dimensional vector space $\mathfrak{m}_{x} / \mathfrak{m}_{x}^{2}$, where $\mathfrak{m}_{x}$ is the maximal ideal of the 
local ring $\mathcal{O}_{x}$ of germs of regular functions near $x$. Since $G \neq S L(i)$ $(i=2,3)$ and $\operatorname{dim} \boldsymbol{G L}\left(\mathfrak{m}_{x} / \mathfrak{m}_{x}^{2}\right)=9, G$ acts on $\mathfrak{m}_{x} / \mathfrak{m}_{x}^{2}$ trivially. Consider the following exact sequence:

$$
0 \longrightarrow \mathfrak{m}_{x} / \mathfrak{m}_{x}^{2} \longrightarrow \mathcal{O}_{x} / \mathfrak{m}_{x}^{2} \longrightarrow \mathcal{O}_{x} / \mathfrak{n}_{x} \longrightarrow 0 .
$$

$G$ acts on $\mathfrak{m}_{x} / \mathfrak{m}_{x}^{2}$ and $\mathcal{O}_{x} / \mathfrak{m}_{x} \simeq k$ trivially so acts trivially on $\mathcal{O}_{x} / \mathfrak{m}_{x}^{2}$ too, since $G$ is linearly reductive. Next, we consider the following exact sequence:

$$
0 \longrightarrow \mathfrak{m}_{x}^{2} / \mathfrak{m}_{x}^{3} \longrightarrow \mathcal{O}_{x} / \mathfrak{m}_{x}^{3} \longrightarrow \mathcal{O}_{x} / \mathfrak{m}_{x}^{2} \longrightarrow 0
$$

Since $G$ acts trivially on $\mathfrak{m}_{x}^{2} / \mathfrak{m}_{x}^{3} \simeq S^{2}\left(\mathfrak{m}_{x} / \mathfrak{m}_{x}^{2}\right)$ and $\mathcal{O}_{x} / \mathfrak{m}_{x}^{2}$, $G$ acts on $\mathcal{O}_{x} / \mathfrak{m}_{x}^{3}$ trivially. By induction, we find that $G$ acts on $\mathcal{O}_{x} / \mathrm{m}_{x}^{n}$ trivially for all $n>0$. So, $G$ acts on the completion $\hat{\mathscr{O}}_{x}$ of $\mathcal{O}_{x}$ trivially, hence trivially on $\mathcal{O}_{x}$. But this implies that $G$ acts on $Z$ itself trivially.

Q.E.D.

Resuming the proof of Theorem 1, we now find $\tilde{X}$ to be $G$-homogeneous, since $\tilde{X}$ has no 1-dimensional or 2-dimensional orbits by Lemma 3 and Lemma 4 , and has no fixed points by Lemma 5 . Since the exceptional locus $E$ of $\pi(\subset \tilde{X})$ is $G$-stable, $E$ must be empty and $\pi$ is an isomorphism. Thus, we conclude that $X$ itself is smooth and $G$-homogeneous.

Now, we will consider the $G$-equivariant contraction maps of $X$. By the same reasoning as in Lemma $4, X$ has an extremal ray $R$. If $f:=$ cont $_{R}: X \rightarrow Y$ is birational, then the exceptional set of $f$ consists of an irreducible divisor $D$ (cf. Mori [11; Theorem (3.3), pp. 146]). Since $f$ is $G$-equivariant, $D$ is $G$-stable which contradicts the $G$-homogeneity of $X$. If $\operatorname{dim}(Y)=1$ or 2 , then $Y$ is also G-homogeneous which contradicts Lemmas 3 and 4. Hence, we conclude that $Y=\operatorname{Spec}(k)$ and $X$ is a Fano threefold of the first kind. [At this point, noting that $X$ is simplyconnected (cf. Iskovskih [7, [8]), we could apply the classification theory of Kähler $C$-spaces (= simply-connected compact Kähler homogeneous manifolds) with $b_{2}=1$, and be done with the proof, assuming $k=C$ (cf. Kobayashi-Ochiai [9]). In this note, however, we persist in the algebrogeometric approach and give a simple proof which depends on the standard properties of Fano threefolds of the first kind.]

Let us, therefore, study the $G$-homogeneous Fano threefolds $X$ of the first kind, i.e., the ones with Picard number $\rho(X)=1$. Referring the reader to Iskovskih's papers $[7,8]$ for basic facts and definitions about Fano threefolds, we shall show that for such $X$ the index, denoted ind $(X)$, 
cannot equal 1 or 2 . Then, ind $(X)=4$ (which means $X \simeq P^{3}$ ) and $\operatorname{ind}(X)$ $=3$ (which means $X \simeq Q_{2}$ ) are the only possible cases.

First, assume that ind $(X)=2$. We use the notations of Table (6.5) in Iskovskih $[8 ; \S 6$, pp. 505], and in the following $H \in \operatorname{Pic}(X)$ stands for the ample generator of $\operatorname{Pic}(X) \simeq Z$. It is easy to see that $H$ is a $G$ linearizable line bundle (cf. Mumford-Fogarty [13; Chapter 1, §3, pp. 30]). Now, in the case (3) of Iskovskih's table, the linear system $|H|$ has a unique base point which must be a fixed point of $G$, a contradiction. In the case (4), $|H|$ has no base points and the associated morphism $h:=$ $\Phi_{|H|}: X \rightarrow \boldsymbol{P}^{3}$ is a double covering with a smooth quartic ramification $S$. Since $h$ is $G$-equivariant, $S$ is $G$-stable. But $\boldsymbol{P}^{3}$ is also $G$-homogeneous, a contradiction. In the case (5), $X$ is a cubic threefold which is not rational (cf. Clemens-Griffiths [3]), a contradiction. In the case (6) (resp. (7)), $h:=\phi_{|H|}: X \rightarrow P^{5}$ (resp. $P^{\bullet}$ ) is a $G$-equivariant closed embedding. Consider $T:=\left\{\right.$ lines in $P^{5}\left(\right.$ resp, $\left.P^{6}\right)$ contained in $\left.X\right\}$. It is known that $T=$ an abelian surface in the case (6) and $T \simeq \boldsymbol{P}^{2}$ in the case (7) (cf. Iskovskih [7; 5 , Remark (5.4), pp. 508]). It is easy to check that $G$ acts on $T$ regularly. Now, from Lemmas 3 and 4 , we know that $G$ acts on $T$ trivially. Hence $G$ fixes every line on $X$, a contradiction.

Next, let us assume that $\operatorname{ind}(X)=1$. Then $|H|$ is base-point free and $h:=\phi_{|H|}$ is described as in one of the following three cases:

(a) $h: X \rightarrow P^{3}$ is a double covering with a sextic ramification.

(b) $h: X \rightarrow Q_{2}\left(\subset P^{4}\right)$ is a double covering with a ramification in a surface of degree 8 .

(c) $h: X \rightarrow \boldsymbol{P}^{g+1}$ is a closed embedding ( $3 \leq g \leq 12, g \neq 11$ ).

In cases (a) and (b), the same argument as the one in the case (4) with ind $(X)=2$ gives a contradiction. In the case (c), it is known that there is a 1-parameter family of lines on $X$ (cf. Iskovskih $[7 ; \S 3]$ ), so that the same argument as in cases (6), (7) with ind $(X)=2$ gives a contradiction. We can now conclude that $X \simeq \boldsymbol{P}^{3}$ or $Q_{2} \subset \boldsymbol{P}^{4}$.

Now, since $\operatorname{dim} \operatorname{Aut}\left(\boldsymbol{P}^{3}\right)=\operatorname{dim} \boldsymbol{P G L}(4)=15$ and $\operatorname{dim} \operatorname{Aut}\left(Q_{2}\right) \leq \operatorname{dim}$ Aut $\left(\boldsymbol{P}^{4}\right)=24$, the dimension counting of simple algebraic groups leaves only the following possibilities:

$$
G=A_{3}, A_{4}, C_{3}, B_{2}, B_{3}, G_{2} .
$$

(Here we are using the standard notations of the corresponding simple Lie algebras.) 
But we can immediately eliminate $C_{3}, B_{3}$ and $G_{2}$ because the minimum dimension of their irreducible representations is 6,7 and 7 respectively (cf. Varadarajan [20; Chap. 4, Exercises]).

Finally, let us eliminate $A_{4}$. Assume $G=S L(5)$, and $G$ must then act on $Q_{2}$ nontrivially, but not on $\boldsymbol{P}^{3}$. Then, as one can readily ascertain, this $G$-action on $\boldsymbol{Q}_{2}$ is the restriction of its action on $\boldsymbol{P}^{4}$, which is induced by the natural 5-dimensional representation $\varphi_{+}$or $\varphi_{-}:=\left(\varphi_{+}^{t}\right)^{-1}$. In either case, $P^{4}$ is a $G$-homogeneous space and does not have any $G$-stable subset other than itself, a contradiction.

Therefore $G$ must be $\boldsymbol{S L}(4)$ or $\boldsymbol{S O}(5)$. In the case $G=\boldsymbol{S O}(5)$, there are a unique 4-dimensional irreducible representation $\gamma_{4}$ and a unique 5dimensional irreducible representation $\gamma_{5}$ up to conjugation (cf. Varadarajan [20; Chapter 4, pp. 394]). We consider $\boldsymbol{P}^{3}$ equipped with the $\boldsymbol{S O}$ (5)-action induced by $\gamma_{4}$. This is the unique $\boldsymbol{S O}(5)$-action on $\boldsymbol{P}^{3}$ up to $\boldsymbol{S O}(5)$-equivariant isomorphisms. The other representation $\gamma_{5}$ is simply the natural 5 -dimensional representation $S O(5) \rightarrow G L(5)$ and, by definition, this action makes a nondegenerate symmetric quadratic form $q$ invariant. We set $Q_{2}=\{q=0\}$ and this $\boldsymbol{S O}(5)$-action on $Q_{2}$ is the unique $\boldsymbol{S O}(5)$-action on $Q_{2}$.

In the case $G=S L(4)$, let $\beta_{+}: S L(4) \rightarrow G L(4)$ be the natural 4-dimensional representation of $S L(4)$ and set $\beta_{-}:=\left(\beta_{+}^{t}\right)^{-1}$. Then $\beta_{+}$and $\beta_{-}$are all and only (mutually inequivalent) 4-dimensional irreducible representations of $\boldsymbol{S L}(4)$. We denote the $\boldsymbol{P}^{3}$ equipped with an $\boldsymbol{S L}(4)$-action induced by $\beta_{+}$ (resp, $\beta_{-}$) by $\boldsymbol{P}_{+}^{3}$ (resp. $\boldsymbol{P}_{-}^{3}$ ). Then $\boldsymbol{P}_{+}^{3}$ and $\boldsymbol{P}_{-}^{3}$ are all the nonisomorphic $\boldsymbol{S L}(4)$-actions on $\boldsymbol{P}^{3}$. It is easy to see that $\boldsymbol{S L}(4)$ cannot act nontrivially on $Q_{2}$.

Q.E.D.

Our Lemmas 3 and 4 above describe all possible closed subgroups of codimension 1 or 2 up to conjugation in simple algebraic groups. At codimension 3 level, combining Theorem 1 with Klein's results contained in Blichfeldt [2] as well as Mabuchi's results [10], we have a complete classification as follows:

CoROllary 6. Let $G$ be a simply-connected, simple algebraic group. Then the following assertions hold:

(1) There exists a closed subgroup $H \subset G$ of codimension 3 if and only if $G=S L(i)(2 \leq i \leq 4)$ or $G=S O(5)$.

(2) The following table gives a complete classification of the closed 
subgroups $H \subset G$ of codimension 3, up to conjugation (or, in other words, the 3-dimensional homogeneous spaces $G / H$ up to G-equivariant isomorphisms):

\begin{tabular}{c|c|c}
\hline$G$ & description of $H$ (or $G / H)$ & $\#$ of conjugacy classes \\
\hline$S L(2)$ & 5 types of finite subgroups [2] & $\infty$ \\
\hline$S L(3)$ & $\begin{array}{l}\text { the dense open orbits of the } \\
\text { following quasi-homogeneous } \\
\text { threefolds of } S L(3): \\
\pm \operatorname{Proj}\left(\mathcal{O}_{\boldsymbol{P}_{2}} \oplus \mathcal{O}_{\boldsymbol{P}^{2}}(-m)\right)(m \geq 1), \\
\pm \operatorname{Proj}\left(T_{P^{2}}\right) .\end{array}$ & $\infty$ \\
\hline$S L(4)$ & $P_{+}^{3}, P_{-}^{3}$ & 2 \\
\hline$S O(5)$ & $P^{3}, Q_{2}$ & 2 \\
\hline
\end{tabular}

Remark 7. In the above table, $T_{P^{2}}$ denotes the tangent bundle of $\boldsymbol{P}^{2}$, and the signature \pm of $\operatorname{Proj}\left(\mathcal{O}_{P^{2}} \oplus \mathcal{O}_{P^{2}}(-m)\right)$ and $\operatorname{Proj}\left(T_{P^{2}}\right)$ in the case $G=S L(3)$ means the same as in the case $G=S L(4)$ (cf. proof of Theorem 1). For the details, see Mabuchi [10] and in the case $G=S L(2)$, see Blichfeldt [2].

\section{REFERENCES}

[1] Bialynicki-Birula, A., Some theorems on actions of algebraic groups, Ann. of Math., 98 (1973), 480-497.

[2] Blichfeldt, H. F., Finite collineation groups, The University of Chicago press, Chicago, 1917.

[ 3 ] Clemens, C. H. and Griffiths, P. H., The intermediate jacobian of the cubic threefold, Ann. of Math., 95 (1972), 281-356.

[4] Dynkin, E. B., Maximal subgroups of the classical groups, Amer. Math. Soc. Transl. Ser. 2,6 (1957), 245-378.

[5] Hironaka, H., Resolution of singularities of an algebraic variety over a field of characteristic 0, Ann. of Math., 79 (1964), I: 109-203, II: 205-326.

[6] Hochschild, P., Basic Theory of Algebraic Groups and Lie Algebras, GTM, 75, Springer-Verlag, New York-Heidelberg-Berlin, 1981.

[ 7 ] Iskovskih, V. A., Fano 3-folds. I, Math. USSR Izvestija, 11 (1977) No. 3, 485-527.

[8] - Fano 3-folds. II, Math. USSR Izvestija, 12 (1978) No. 3, 469-506.

[9] Kobayashi, S. and Ochiai, T., Compact homogeneous complex manifolds with positive tangent bundle, Differential Geometry in honor of $\mathrm{K}$. Yano, Kinokuniya, Tokyo, 1972, 221-232.

[10] Mabuchi, T., On the classification of essentially effective $S L(n, C)$-actions on algebraic $n$-folds, Osaka J. Math., 16, No. 3 (1979), 745-759. 
[11] Mori, S., Threefolds whose canonical bundles are not numerically effective, Ann. of Math., 116 (1982), 133-176.

[12] Mukai, S. and Umemura, H., Minimal Rational Threefolds, Lec. Notes in Math., 1016, Springer-Verlag, Berlin-Heidelberg-New York, 1983, 490-518.

[13] Mumford, D. and Fogarty, J., Geometric Invariant Theory, A Series of Modern Surveys in Mathematics, Springer-Verlag, Berlin Heidelberg New York, 1982 (Second Enlarged Edition).

[14] Nakano, T., On equivariant completions 3-dimensional homogeneous spaces of $S L(2, C)$, to appear in Japanese J. of Math., Vol. 15, No. 2, 1989.

[15] Sumihiro, H., Equivariant completion II, J. Math. Kyoto Univ., 15-3 (1975), 573605.

[16] Umemura, H., Sur les sous-groupes algébriques primitifs du groupe de Cremona à Trois variables, Nagoya Math. J., 79 (1980), 47-67.

[17] - Maximal algebraic subgroups of the Cremona group of three variables, Nagoya Math. J., 87 (1982), 59-78.

[18] - On the maximal connected algebraic subgroups of the Cremona group II, Advanced Studies in Pure Math., 6 (1985), Algebraic Groups and Related Topics, 349-436.

[19] - Minimal rational threefolds II, Nagoya Math. J., 110 (1988), 15-80.

[20] Varadarajan, V. S., Lie Groups, Lie Algebras and their Representations, GTM 102, Springer-Verlag, New York•Berlin·Heidelberg•Tokyo, 1984 (Springer Edition).

Department of Mathematical Sciences

Tokyo Denki University

Hatoyama-machi, Hiki-gun

Saitama-ken, 350-03 Japan 